\title{
DAVID HUME, LA IMPOSIBILIDAD DE UN PROGRESO EN LOS SENTIMIENTOS MORALES
}

\author{
José Reinel Sánchez \\ Universidad del Quindío \\ rsanchez@uniquindio.edu.co
}

\begin{abstract}
Resumen
El artículo aborda los alcances de la propuesta moral de David Hume, específicamente la idea de que, ante la imposibilidad de realizar propósitos morales sinceros, el camino que queda para el individuo es fingir que dichos sentimientos se han adquirido. Tal actitud es vista por el autor como favorable para las buenas relaciones sociales y para la construcción de acuerdos de convivencia entre individuos egoístas posesivos. Sin embargo, dado el radical determinismo de la mente concebido por Hume, tal esfuerzo mostraría la intención por coadyuvar a soluciones pacíficas al problema de la insociabilidad, pero no tendría efectos reales sobre las pasiones originales de los individuos.
\end{abstract}

PalABRAS ClaVE: moralidad, la mente como un teatro, determinismo, simpatía, progreso de los sentimientos, fingir.

\section{Abstract}

This essay brings up the extent of David Hume's moral proposal; specifically, the idea that facing the impossibility of accomplish sincere moral aims the individual is left to feign that such sentiments have been acquired. Such attitude is seen by the philosopher as favourable for proper social relations and for the shaping of coexistence agreements amongst possessive selfish individuals. Nevertheless, given such radical determinism of the mind conceived by Hume, the effort would remark only the attempts to team up with for reaching peaceful solutions for the problems of unsociability but would not have real effects over the original passions of the individuals.

KEY WORDS: morality, the mind as a theatre, determinism, sympathy, Progress of sentiments, feign.

RA David Hume, un filósofo de estirpe hobbesiana, dejó planteado en su Treatise of Human Nature (1739) ${ }^{1}$ algunas dificultades insuperables en la realización de los proyectos morales. Estas dificultades son propias de su percepción de una naturaleza humana fundada en el determinismo y en una modalidad radical de egoísmo.

Se utilizará $A$ Treatise of Human Nature edited, with an analytical index, by L. A. SelbyBigge, M. A. Oxford: Clarendon Press, 1896. Se citará así (T. pág.). 
Sin embargo, el autor afirma la necesidad de que los individuos adquieran unas reglas morales eficientes para la paz y la concordia. Si bien Hume sostiene a lo largo de la obra que estos sentimientos pueden generarse, indicando la existencia de un progreso en los sentimientos morales. Sin embargo, a pesar de los esfuerzos del autor por mostrar que tal progreso es posible, debe apelar al fingimiento o la simulación de dichos sentimientos, dejando entrever un nivel de frustración respecto de los alcances de su teoría moral misma.

Si bien Hume no parte de los presupuestos básicos de la teoría política de Hobbes como, por ejemplo, el estado de naturaleza, pues lo considera analíticamente no pertinente e históricamente inexistente, el panorama que puede describirse de las relaciones entre individuos posesivos (MacPherson 1962) es de deseo de gloria, desconfianza y competitividad - esta incluye hostilidad- lo cual puede considerarse como hobbesianismo puro. A juicio de Hume, la estabilidad de la sociedad exige una disposición mental que permita que individuos de esta clase desvíen sus intereses egoístas y redirijan sus sentimientos para favorecer la vida social y esto solo es posible mediante la adquisición de sentimientos de honestidad y justicia.

La mayor dificultad estriba en que los fundamentos de la teoría moral humeana son presentados en perspectiva mecánica: los movimientos de los procesos mentales se rigen por los mismos principios que rigen los movimientos de los cuerpos físicos; en tales procesos impera el determinismo de la naturaleza humana. Para mi propósito solo referiré tres argumentos que resultan ser los más relevantes en la descripción de las imposibilidades de las acciones morales proyectadas:

A1. Determinismo: no se puede derivar un debe de un es (T. 469).

A2. El sentimentalismo: un agente solo puede hacer lo que le mandan sus sentimientos (T. 415).

A3. Individualismo posesivo: el agente solo actúa motivado por la ventaja económica que pueda obtener de su acción (T. 491-492).

Desde estos argumentos puede deducirse que prima el egoísmo en grados muy altos y que, por esa razón, Hume debe contemplar la idea de que, ante la imposibilidad de realizar propósitos morales, el individuo, al menos, debe forzarse a fingir. Expresamente lo dice así:

[T] he expression of a resolution is not commonly suppos'd to be obligatory; and we cannot readily conceive how the making use of a certain form of words shou'd be able to cause any material difference. Here, therefore, we feign a new act of the mind, which we call the willing an obligation; and on this we suppose the morality to depend (Hume 1978, p. 523).

Esto es, que ante la exigencia de los otros, de hacer que el individuo sea aquello que no es, el agente haga pública una actitud como si hubiera alcanzado sentimientos morales loables. Esta salida da margen para considerar que los propósitos para alcanzar un mejor mundo, por la vía de la adquisición de sentimientos morales, podría ser evaluado 
de antemano como una frustración segura y, por tanto, como un esfuerzo inútil hecho por el agente, lo cual resulta coherente con lo que referí como el argumento (A1); el individuo viviría inmerso en el 'cuento fabuloso' de ser moralmente excelso. El presente trabajo se propone presentar la teoría de los sentimientos morales de Hume y sacará algunas conclusiones sobre el verdadero impacto de dichos sentimientos en la conducta individual.

Vistas las cosas de esta manera, la primera pregunta que surge es ¿cómo es posible que individuos naturalmente orgullosos, competitivos y hostiles puedan ser moralmente buenos y/o mantener su palabra fundada en virtudes morales? Si los rasgos de comportamiento del individuo posesivo son perpetuos no podría haber momentos de confianza ni acercamiento con fines de puesta en acuerdo con otros sobre unas normas generales que han de ser vistas como lo excelente digno de ser vivido. Según Hume, la necesidad de vivir cómoda y tranquilamente es uno de los motivos que facilitan el surgimiento de la justicia (T. 485). Tal argumento finalmente no es muy convincente porque, dada la dimensión autocentrada y egoísta de los seres humanos, no podría esperarse que el deseo de bienestar fuera la causa de la vida humana moralizada. Hume considera que la vida moral proporcionaría seguridad y tranquilidad, pero realmente no podría ser así pues los seres humanos son exitosos (A3) más por el grado de sagacidad y prudencia que alcancen que por su capacidad de moralidad (T. 610) y, además, porque el deseo de éxito motiva acciones temerarias ${ }^{2}$.

A mi parecer, Hume es consciente de este fracaso y, por esa razón, finalizando el Tratado se aventura a redirigir su aspiración de realización del proyecto moral al campo de lo fingido (T. 523), o sea, de lo no auténtico; lo que finalmente propone Hume en su teoría moral es que hemos de fingir que realizamos nuestras proyecciones morales porque estamos incapacitados para realizarlas sinceramente; según Hume, el efecto será psicológico, positivo para la vida pública, pero esto no tiene un verdadero impacto en la personalidad del individuo.

La exposición será dividida como sigue: en el primer aparte presento dos escollos para la realización de la moralidad: el determinismo fisicalista y el individualismo posesivo; en el segundo aparte refiero una teoría de la moral derivada del dilema del prisionero iterado (DPI); en un tercer aparte desarrollo la que denomino la experiencia de la moralidad; $y$, finamente presento lo que denomino la frustración de no alcanzar la vida moral.

Esta preocupación es manifiesta tanto en Hobbes como en Hume. El primero plantea el problema del 'tonto' (Hobbes 1996/1651, p. 111), el segundo lo plantea en el 'Sensible knave' (Hume 1930, p. 122) 


\section{Escollos para la realización de la moralidad}

\section{Primer escollo: el determinismo fisicalista}

Hume es consciente de que la conducta cooperativa de los individuos posesivos constituye un elemento importante en la construcción y permanencia de la vida civilizada e industriosa, aunque también advierte que los individuos solo pueden proponerse hacer lo que su naturaleza les determina.

Este problema será planteado desde dos aspectos del argumento (A1). El primero afirma que no se puede derivar un debe de un es; la diferencia estriba en que el es describe un estado de cosas mientras que el debe plantea una prescripción. Si se afirma que un individuo es egoísta, competitivo y agresivo no podrá afirmarse que tal individuo pueda, porque él se auto-prescriba u otra persona le prescriba ser otra cosa (ser un ángel) o ser ella misma de otro modo (ser un vampiro bueno), por ejemplo; percibido así el asunto, las expectativas de influencia de la auto-prescripción moral se pueden reducir a nada. El segundo aspecto muy vinculado con el anterior es el siguiente: Hume da un alto valor de eficiencia a los hábitos y las costumbres $(T .86,424)$ como contenido cognitivo de la mente tanto en lo que se refiriere a los comportamientos individuales como a las dinámicas de los movimientos sociales: los seres humanos actúan dominados por los surcos que los hábitos y las costumbres crean en las mentes de los seres humanos: las costumbres dan un sesgo a la imaginación (T. 148).

En consecuencia, la vía humeana no es alentadora para quienes aspiran a que un individuo realice un determinado comportamiento excelente desde la consideración de que es bueno o mejor para la vida humana porque los movimientos mecánicos de las operaciones internas de la mente tienen un peso enorme en la configuración del carácter de las personas; en la definición de sus sentimientos y en la relación de tales personas con otras. En términos sociales, la proyección de una vida excelente, digna de ser vivida e imitada a partir del deseo de negar la hostilidad natural tiene la bondad de sugerir un esfuerzo por corregir nuestras actitudes pero no podría alterar, para nada, la naturaleza humana. ¿Para qué, entonces, pensar la moralidad? Si no puede ser eficaz para la acción ¿Para qué hablar de moralidad? Con cierto optimismo puede decirse que (para Hume) las normas morales son funcionalmente inútiles, en lo que respecta a los alcances proposicionales o intencionales, pero que ideológicamente son valiosas.

\section{Segundo escollo: el sentimentalismo del individualismo posesivo}

El campo de la mente que trata las acciones morales es el de las impresiones de reflexión, campo de las ideas; este se ocupa exclusivamente de las emociones y las pasiones (T. 8). De esta facultad mental surgen los motivos para actuar, y siempre estará subsumida a 
la iniciativa de las pasiones (A2) y a la utilidad ${ }^{3}$ que el objetivo de la acción representa para el individuo (A3). El autor reporta un numeroso grupo de pasiones que configuran a un tipo de ser humano y con él a un modelo de sociedad, configurando la mentalidad del individuo posesivo ${ }^{4}$; de ellas solo resaltaré dos que tienen un fuerte impacto en la radical concepción posesiva de la vida humana:

\section{El deseo de ganancia:}

Tis certain, that no affection of the human mind has both a sufficient force, and a proper direction to counterbalance the love of gain, and render men fit members of society, by making them abstain from the possessions of others ( $T$ 492);

\section{El egoísmo:}

We make allowance for a certain degree of selfishness in men; because we know it to be inseparable from human nature, and inherent in our frame and constitution $(T 583)^{5}$.

Para que un individuo, con esta configuración sentimental natural, pueda llevar una vida agradable en unión con otros, deberá atender, según Hume, a un grupo de virtudes de origen artificial con funciones estrictas de cohesión social y que haría de la moralidad un aspecto de la vida humana no solipsista, agradable y normatizada; ellas son la "lealtad al gobierno, las leyes de naciones, la modestia y las buenas maneras" (T. 577). Hume está convencido de que es necesario romper la cadena causal egoístico-sentimental negativa para la vida social: los individuos humanos tienen que forzarse a establecer vínculos de confianza y de respeto, esto solo puede ser posible si se logra reducir el orgullo y la agresión a los otros, y asumiendo una actitud cooperativa; ésta condiciona un trato moderado y respetuoso a los otros no allegados. Esto lo percibe Hume como posible y factualmente puede mostrarse: la sociedad humana existe. La pregunta que

3 No por esto hay que afirmar que Hume sea utilitarista; el concepto como es referido hoy está basado en una de las tesis de J. Bentham: la felicidad del mayor número (Bentham 2008). Para otros aspectos ver el trabajo de Ronald J. Glossop “Is Hume a 'Classical Utilitarian'?” (1976).

4 El vínculo entre Hobbes y Hume es grande, muy específicamente desde la idea de la naturaleza autocentrada y egoísta de los individuos humanos. Dos conceptos pueden aplicarse a esta conducta del individuo humeano: 1. El individuo posesivo (MacPherson 1962); 2. El egoísta incompleto (Gauthier 1990) Si bien Hume trata de unir moral y ventaja, el experimento solo puede evaluarse como positivo en la perspectiva del comportamiento económico. Pero en perspectiva moral es insuficiente y demasiado provisional. Tampoco aplica para vincularlo a una corriente utilitarista.

$5 \quad$ Hay, además, otras pasiones: el amor propio ( $T 480)$, la codicia $(T 439,487)$, la parcialidad ( $T 321)$, el orgullo (T276, 390); estas cuatro pasiones están muy vinculadas con la psicología del individuo posesivo. 
surge es ¿Tal obligación de trato a los otros es posible de manera auténtica? ¿Cómo es que individuos orgullosos y maximizadores económicos pueden llevar una vida bien relacionada con los otros? La necesidad o el miedo a la muerte, siendo buenos argumentos para la socialidad, pueden resultar respuestas ligeras e insuficientes dada la fuerza que emiten las pasiones egoístas. Un proyecto racionalista de construcción de la sociedad fracasa porque la fuerza de las pasiones es tan enorme que la razón, inerte por naturaleza ( $T .459)$ está incapacitada para motivar acciones auténticas para la obtención de buenas relaciones con los otros.

La fuerza ineludible de las pasiones naturales, de los hábitos personales y de las costumbres sociales viene a delimitar los alcances de los propósitos de los individuos, quedando, únicamente un mundo inalterable compuesto de sujetos cuya tendencia natural es excluirse mutuamente. Sin embargo, el mundo social fáctico muestra la existencia de esas relaciones; y tal existencia, percibida por Hume, es apoyada por la idea de que existe un progreso cognitivo y pasional en la vida humana; con cierto asombro uno puede sentir que su propuesta nos sugiere una idea de la evolución de la sociedad y con ella la posibilidad de considerar que hay un progreso en los sentimientos que se puede percibir en que los individuos adquieren un tipo especial de sentimiento al que se denomina moral porque implica la adquisición de una conducta excelente digna de ser alabada; esta aspiración podría ser coherente con un proyecto de sociabilidad ilustrada pero incoherente con los argumentos teóricos ya señalados.

\section{Una teoría de la moral derivada del dilema del prisionero iterado}

Hume, como Hobbes, pudo salir airoso del problema si hubiera considerado que los individuos tienen la facultad de la razón, la cual tiene la potestad de indicar cómo actuar de la mejor manera. Para Hobbes, las leyes de naturaleza, resultado del uso práctico de la razón, sugieren actuar de manera eficiente (Hobbes, 1996/1651) a favor de la conservación de la vida. Sin embargo, Hume considera que la razón es inerte o, en su defecto, que es sierva o esclava de las pasiones $(T .415)^{6}$. La razón puede indicar la salida lógicamente correcta pero es incompetente para ser propositiva-intencional $y$, por tanto, ejecutar lo que sugiere porque lo correcto que le compete está limitado a asuntos formales relacionados con el descubrimiento de la verdad, no con la rectitud de asuntos prácticos (T. xv). En este aspecto, no poder derivar un debe de un es, solo dice

$6 \quad$ El problema es un poco más complicado. La tesis fundamental aquí es que a la razón solo le corresponden operaciones lógico-formales concernientes a la verdad: la razón, por sí misma no motiva a actuar. El mejor ejemplo es su afirmación que no es contrario a la razón preferir la destrucción total del mundo al rasguño de un dedo (Cfr. T. 416). Hume reconoce que la razón sí tiene influencia, pero su influencia es muy discreta y no es decisiva. Sobre este tema, ver Thomas Huff "Self-Interest and benevolence in Hume's Account of Moral Obligation" (1972). 
que no es posible concebir modificación alguna de la conducta a partir de proyectos preconcebidos, así sean bien planeados y bien intencionados; el refrán "Por mucho que madrugue no amanecerá más temprano" puede ilustrarnos mejor la idea: no está en nuestras manos determinar el momento del amanecer, así sea deseado vehementemente por el individuo. Lo que nos está diciendo realmente Hume es que hay que considerar que los seres humanos están dominados por pasiones egoístas, las cuales solo son sofrenadas por la influencia negativa de los otros sobre el agente, pero a pesar de esta influencia dicho individuo será siempre el mismo. Un ejemplo, de obligatoria citación, es el de dos granjeros ( $T$. 520) quienes han de mantener el compromiso de cooperación por el miedo de las retaliaciones futuras que recíprocamente se podrían producir.

Entonces, ¿cómo hablar de progreso en los sentimientos morales? Cuando Hume lo hace en el Tratado se refiere a la unión simpatética que alcanzan los individuos a favor del interés público (T. 500) En buena retórica dice que los individuos de una nación crean una identidad no por el terreno geográfico que ocupan sino por la simpatía. Por la influencia de tal principio ellos convergen en el 'interés público', y este es la medida del mencionado progreso. Se marca así la diferencia entre el comportamiento del individuo autocentrado y el de aquel que piensa en el 'interés público', entre egoísta puro y el egoísta simpático. La industria, comercio y cultura han propiciado un mejoramiento en la calidad de vida y pueden llevarnos a pensar que cuando Hume habla de progreso se refiere al perfeccionamiento de la vida humana, pero esto es equivocado. La equivocación estriba en dos aspectos: primero, las modificaciones en las emociones que favorecen la vida social no son el resultado del surgimiento de la civilización sino al revés, esto solo es posible porque ya las personas habían adquirido maneras de conversar y de tratarse bien; segundo, no hay modificación sustantiva de la naturaleza humana.

Hume piensa que no se puede negar que con el uso de actividades civilizatorias las expectativas de vida humana hayan mejorado; tal percepción de la vida corregida puede percibirse en el ámbito moral por la práctica de las buenas maneras, del refinamiento del gusto y de la justicia.

\section{La simpatía y la consolidación de las relaciones con otros}

La simpatía refiere a un principio psicológico físico-mecánico que propicia la comunicación de sentimientos y que facilita una relación relativamente diáfana entre personas que comparten de manera auténtica sentimientos y actúan en conformidad. Se trata de la simpatía, la cual genera la uniformidad de 'humores' o temperamentos propia de las agrupaciones humanas como el Estado, él lo expresa de esta manera: "To this principle (sympathy) we ought to ascribe the great uniformity we may observe in the humours and turn of thinking of those of the same nation" (T. 316).

A esta convergencia de sentimientos, conformando una esfera pública, es a lo que Hume llama progreso de los sentimientos (T. 500). El concepto "progreso" pues hace referencia a la forma como los individuos se van integrando interesada y sentimentalmente a las diversas esferas sociales permitiendo consolidar grupos de beneficio común. Sin embargo, dicho concepto es difícil de captar por los objetos a 
los cuales Hume atribuye esa cualidad y por el sentido en que lo usa: él utiliza el verbo progresar para referirse a un avance del pensamiento ( $T$. 221), de la imaginación ( $T$. 204), del quilo (T. 325), de la filosofía (T. 332), etc. Con la idea de progreso, Hume no hace referencia al mejoramiento incesante en la historia humana, a su evolución o a su perfeccionamiento, como lo afirma K. Marx. Más bien, en este campo nuestro autor es un completo conservador pues (1) a su juicio no habría sino dos 'estadios' históricos: el presocial y el social. El primero, muy corto, se caracteriza por el primado del egoísmo individual y tribal; el segundo se caracteriza porque la sociedad y la clase humana viven bajo la directriz de los principios de justicia, pero sin la superación del comportamiento presocial. (2) El ser humano siempre será el mismo, estará siempre regido por los mismos principios y las mismas pasiones; este es el sentido del argumento (A1). El autor expresamente afirma que los hombres siempre serán egoístas, ambiciosos. A la ya referida afirmación de la página ( $T$. 583) se puede radicalizar el argumento pues, además de estar determinados a ser lo que son, los seres humanos son egoístas miopes y sólo prefieren el bien más cercano al más remoto ( $T$. 419).

Si bien es cierto que Hume se apoya en la simpatía como principio natural que posibilita la socialidad entre individuos no familiares, es necesario tener presente que lo que es realmente eficiente para el propósito moral es la educación (T. 479), institución que adoctrina a los individuos que caen bajo su tutela. Hume considera que mediante un entrenamiento adecuado se puedan refinar las pasiones egoístas originales, moralizarlas, permitiendo que el individuo redireccione sus emociones (A2) hacia el logro de la acción colectiva y del interés público. Esto quiere decir que mediante cierto proceso mental, las impresiones han de abandonar la fase de sensualismo simple y directo e instalarse como incitadores a la acción generando motivadores volitivos de segundo orden $\left(D^{2}\right)$; esto es, sentimientos creados artificialmente, pero asumidos como propios (por ejemplo: "Yo deseo adquirir el deseo de ser un individuo moralmente ejemplar y ser aplaudido por los otros"7.

$7 \quad$ El tema de los deseos de segundo orden está referenciado por Harry Frankfurt de la siguiente manera: "Besides wanting and choosing and being moved to do this or that, men also want to have (or not to have) certain desires and motives. They are capable of wanting to be differenct, in thier preferences and purposes, from they are" (Frankfurt 1988, p. 12). El problema que puedo percibir en esta descripción es que estos deseos ( $\left.D^{2}\right)$ pueden surgir de expectativas variadas y absolutamente unipersonales (por ejemplo, yo deseo desear ser poeta, o ser vegetariano). El planteamiento de Hume es que la moral es una relación entre un sujeto con un agente externo: "First, As moral good and evil belong only to the actions of the mind, and are deriv'd from our situation with regard to external objects" (T. 464), la exigencia es que para que un deseo sea moral tal deseo moral debe ajustarse a los sentimientos de otros. Una variable ofrecida por David Lewis que resulta más relevante porque implica que el individuo debe coordinar sus deseos con los deseos de otros, involucrando la necesidad de que los deseos que desea $\left(\mathrm{D}^{2}\right)$ el individuo se ajusten a lo que otros esperan que él finalmente haga: "I desire that I go there on condition that you Will go there" (Lewis 1996, p. 29). 
Resumiendo parcialmente, consideremos que en la teoría de la acción de Hume: (1) hay determinismo en la trayectoria de la mente que impide traspasar la barrera del es de la naturaleza humana egoísta; 2) que es posible establecer vínculos con personas extrañas rompiendo el egoísmo; y (3) también que es posible adquirir sentimientos morales hacia algo tan vago como el interés público; esto último solo puede ser entendido como un apaciguamiento del egoísmo por la influencia de la simpatía pero, a mi juicio, es más por un adoctrinamiento ejercido por la educación pública y el artificio de los políticos. El problema que viene a continuación es la consideración de la posibilidad de alcanzar tales deseos $\left(\mathrm{D}^{2}\right)$ y de actuar bajo la dirección de ellos.

\section{2. ¿El egoísmo dominado?}

El problema en torno a la posible realización de nuestros sentimientos morales tiene por trasfondo una teoría de la mente. A juicio de nuestro autor, la mente tiene dos facultades en las que se albergan nuestras percepciones: la memoria y la imaginación. A la memoria corresponde el dominio de eventos que ya han sido vividos. Hume se refiere a eventos que por su vivencia repetida pueden conformar la idea de uniformidad: son series de percepciones uniformes y reiteradas característica que les confieren la fuerza y vivacidad suficientes para recordar los eventos en el orden en que fueron percibidos (cfr. T9); esto tendría mucho valor para la construcción de la mentalidad de un jugador contextualizado en el (DPI) quien debe revivir sus experiencias negativas con otros jugadores para controlar sus pasiones egoístas-posesivas (T. 490). La "imaginación" es referida como la existencia y el manejo de las ideas "débiles y lánguidas" en la mente, motivo por el cual ésta no puede retenerlas de manera uniforme por mucho tiempo (Cfr. $T, 9)$. La imaginación juega un rol importante en la vida humana; gracias a esta facultad es que el ser humano puede proyectarse en un ambiente de percepciones no vividas directamente; por esta facultad es que el ser humano puede anticipar eventos, en esta facultad se alojan las proyecciones morales. El problema es que la imaginación recrea un mundo moralmente deseable a la manera de un superyó, pero por definición es ineficiente para la vida práctica.

La admisión de esta última percepción de la vida humana nos pone en una fuerte tensión en lo que respecta a nuestra naturaleza asocial y las posibilidades de convivencia pacífica: las guerras, las vendettas, las disputas intergeneracionales y otras riñas permanentes, la larga vida infantil autocentrada y autorreferida nos pueden indicar que la presencia de esas pasiones egoístas tienen una influencia perenne en la formación del individuo y confirmar que la paz podría ser considerada como una quimera.

Además surge un problema adicional ¿Quién genera las normas morales? Las opciones son dos y poco sólidas en relación con el objetivo de formar individuos con capacidad de auto-restringir sus pasiones egoístas para favorecer la vida social. Por un lado, fundamentar la vida social en el cumplimiento de reglas de carácter heterónomo siguiendo el modelo de Hobbes de un soberano con carácter de juez que constriña a los individuos a vivir de determinada manera (Leviathan XVI), puede interpretarse como una solución airosa pero débil. Tal salida es en falso en cuanto que si se pierde de vista al juez, ejecutor de la ley, se pierde la capacidad de constricción, por tanto, el individuo 
puede realizarse egoístamente si la ocasión lo permite, ya con preocupación lo planteó el mismo autor al presentar el problema del tonto. El tonto siempre estará tentado a sacarle provecho al descuido o a la confianza de los otros. Por otro lado, si se lograra introducir un grupo de normas autoimpuestas de restricción de carácter moral -tal como el kantismo las concibe-, las garantías de la existencia de la cooperación social serían mayores, pero esto solo es cierto en parte, pues la entrega del honesto puede concluir en torpeza, puede quedar expuesto a las demandas de los más astutos y bribones.

\section{Una teoría de la moral derivada del dilema del prisionero iterado}

La esperanza de conformar sentimientos morales parece darla la sombra del miedo a la retaliación futura. A lo largo del Tratado, David Hume nos da a entender que la mente percibe las cosas por gradaciones. Estas pertenecen a la naturaleza de las cosas y son reales, no son meras ficciones mentales. La parte a la que me referiré aquí es a la vida humana y corresponde al paso (i) del individuo psicológicamente auto-centrado que busca solo el bien propio y el de los suyos a (ii) un estado en el que este mismo individuo psicológicamente se ha habilitado para conformar un cuerpo común con otros en una unidad empática de sentimientos que le permitirá obrar con otros como un agente colectivo.

El proceso de 6 estadios podría ser descrito de esta manera:

1) En el estadio presocial el individuo es egoísta posesivo; 2) sin embargo, el individuo humano no es como lo osos que viven solos en su vida adulta. Posee elementos que le permiten socializarse con los integrantes de su familia y los conocidos. Esta condición le permite ser naturalmente benevolente hacia algunos seres humanos, aunque tal sentimiento esté circunscrito únicamente a los suyos. 3) Desde el interior de la familia, aprende a reconocer y a valorar la asistencia que otra persona pueda prestarle para alcanzar los frutos de su trabajo con algunos rudimentos de justicia. En la familia aprende a valorar las ventajas de ser honesto. 4) El mismo fin tiene la instrucción pública a cargo del Estado. Esta reforzará el espíritu cooperativo. Además, el Estado tiene la potestad de facto de castigar a quien falte a la convención de la justicia. 5) La honestidad se alcanza cuando el individuo sienta remordimiento por romper la norma de la justicia; y 6) El sentimiento de honestidad compartido posibilita la existencia de ese bien público.

El aumento de los grados de los sentimientos que van del 'color' más pálido (1 y 2) a un color más fuerte y vivaz (4) con relación a un ambiente social ordenado y eficiente, es lo que muestra ese supuesto progreso de los sentimientos. Sin embargo, a pesar de la fuerte influencia de socialización que recibe un individuo en las fases (3) y (4) la máxima estabilidad de la sociedad que se alcanzaría a este nivel solo es posible bajo las reglas del DPI. Esta situación, entre negociantes, manifiesta un aparente resultado equilibrado por la sombra del futuro (Axelrod 1984). La teoría moral parece terminar fundada en la capacidad de retaliación recíproca entre negociantes. Este es el sentido de entender el asunto a la drástica manera de Hume. Un mal negocio implica que uno de los agentes participantes en un negocio puede perder la 'confianza' y la 'seguridad' de 
que lo que le pertenece por la convención de la propiedad le será respetado; miremos el caso de los dos granjeros:

Your corn is ripe today; mine will be so tomorrow. 'Tis profitable for us both, that I shou'd labour with you today, and that you shou'd aid me tomorrow. I have no kindness for you, and know you have as little for me. I will not, therefore, take any pains upon your account; and shou'd I labour with you upon my own account, in expectation of a return, I know I shou'd be disappointed, and that I shou'd in vain depend upon your gratitude. Here then I leave you to labour alone: You treat me in the same manner. The seasons change; and both of us lose our harvests for want of mutual confidence and security (Hume 1978, p. 520) ${ }^{8}$.

Empero, justamente, aquí puede estar la debilidad de esta vía economicista humeana, pues finalmente hay que reconocer que no hay confianza plena ni mucho menos seguridad porque obrar racionalmente es una de las condiciones del éxito, pero obrar irracionalmente también ${ }^{9}$. Es apenas dudoso que en el momento menos esperado, dada la condición referida en (A1) y (A3), uno de los jugadores, astuto y temerario, planee que a la $n$ jugada traicionará en el juego para quedarse con la posible mejor paga para él, sin importarle la suerte del otro. La mejor paga neta posible que pueda alcanzar un individuo se hace a expensas de timar a otro porque es una ganancia que no será compartida, esa es la ley del pícaro pero que se ajusta al carácter egoísta y ambicioso que Hume atribuye a los seres humanos. El DPI es una muestra óptima de que el juego limpio paga, pero paga mucho menos de lo que un bribón ambicioso espera y, desde esa perspectiva, la tentación de abandonarlo siempre estará presente.

\section{La experiencia de la moralidad como ficción}

Para estabilizar las relaciones comerciales, Hume exige un poco más que el resentimiento y la capacidad de retaliación de la persona ofendida, entendidos como pasiones que equilibrarían las relaciones sociales. Una mala consecuencia es que entre venganza y venganza se llega, fácilmente, a la conclusión de que los hechos de fuerza son la única garantía de proteger lo que se ha ganado con 'la laboriosidad y la fortuna', esto es, se llega a la situación hobbesiana del estado de naturaleza, la cual es semejante

Cursivas añadidas.

La condición para que el DPI funcione bajo los parámetros de la cooperación equitativa es posible si la sombra del futuro es tan amplia que posibilite encuentros entre agentes:

For cooperation to prove stable, the future must have a sufficiently large shadow. This means that the importance of the next encounter between the same two individuals must be great enough to make defection an unprofitable strategy. It requires that the players have a large enough chance of meeting again and that they do not discount the significance of their next meeting too greatly (Axelrod 1984). 
a la del Dilema del Prisionero (DP). Hume exige que también haya ese sentimiento moral llamado honestidad. Este corresponde al estadio (5); la honestidad solo es posible si se logra conformar un sistema de virtudes que haga que el individuo sienta remordimiento cuando quebrante la norma de la justicia. Hume la refiere como una pena que se inflige una persona a sí misma, ésta tiene su sentido socialmente positivo, pues el remordimiento surge por 'el crimen cometido' (Cfr. T 376). Sin embargo, a mi parecer, la presunción de que el sentimiento de honestidad reforzaría ese equilibrio alcanzado por el DPI también es muy limitada y tiene una gran desventaja para el jugador honesto; el jugador honesto tendrá que estar siempre vigilante de lo que el otro haga o deje de hacer. Si bien una persona honesta podría saber a ciencia cierta que su vida se rige por el sentimiento que favorece el respeto al otro y, por ende, la armonía social, esa misma persona no podría tener esa misma certidumbre sobre las verdaderas intenciones de los otros, excepto presumir su honradez por su conducta uniforme, por su comportamiento en el trato iterado. Ahora bien, es la identidad en el sentimiento de honestidad lo que posibilita la existencia de ese bien público (6). No es, entonces, la capacidad de cooperar, per se, la que conformaría el agente colectivo. Aquí tendríamos los seis matices o estadios que podrían darse en dicho progreso de los sentimientos en el que el sentimiento más vivaz debería corresponder al sentimiento de honestidad, favorable para la vida pública. Pero aquí hay algo nuevo, quien se precie de honesto debe serlo auténticamente: no importa la situación económica del virtuoso: la virtud en andrajos sigue siendo virtud (Cfr. T. 548).

Ahora bien, un nuevo problema, sumado a los ya advertidos del determinismo de la conducta del individuo posesivo, es que Hume está convencido de que lo que la persona exterioriza a los otros es realmente efecto de su sentir. Uno puede pensar, además, que ingenuamente el autor cree que la simpatía es el mecanismo que permite a los individuos comunicarse e interpretar de manera adecuada los sentimientos sinceros de los otros, pues considera que las acciones de las personas están mostrando su verdadero carácter (T.317), sin considerar que una de las habilidades del bribón es que puede representar, como en el teatro, un rol que no es el suyo ${ }^{10}$.

10 Resulta importante en este tema la obra de Paul Ekman. Ekman estudia los gestos, su relación con el comportamiento y la posible universalidad de estos. En "Why Dont We Catch Liars" (1996), "A few can Catch a Liar" (1999) y "Lie Detection And Language Comprehension" (2000), Ekman muestra no solo la gran dificultad que tiene la mayoría de la gente para 'agarrar' a los mentirosos (Ekman 1996) sino, al igual que Hume, reconoce la importancia de cierto tipo de mentira en la buena marcha de las relaciones sociales (Ekman 1996). Ekman aborda el problema planteado por Hume: ¿Cómo compartir e identificar sentimientos socializantes con los extraños en las sociedades modernas si la mala reputación del bellaco deja de ser el arma del honesto?

In modern industrial societies (...) The opportunities for lying are plentiful, privacy is easy to achieve, there are many closed doors. When caught, the social consequences need not be disastrous, for one can change jobs, change epouses, change villes. A damaged reputation need not follow you (Ekman 1996, p. 811). 
Lo que Hume quiere dar a entender es que todo lo que ilustra el DPI, como una salida airosa al DP, podría ser inútil si esta persona es incapaz de comprender que hay aquel bien superior llamado bien público, y no ha deseado desear tal sentimiento $\left(\mathrm{D}^{2}\right)$ que facilite la creencia sincera en las ventajas que ofrece este espacio para la vida humana.

Hume cree que la perfecta vida en comunidad solo está garantizada por el sentimiento moral adquirido a lo largo de la vida, sumado a los vínculos diáfanos entre personas que la simpatía proporciona. Esa vida en común se alcanza por la práctica de las virtudes artificiales que permanentemente están suavizando las asperezas del autocentramiento producto de las pasiones naturales. El caso especial lo merecen las "buenas maneras". Por buenas maneras podemos nombrar un comportamiento cortés, moderado y buena capacidad de conversación que tienen un individuo para solucionar los problemas que las relaciones con los otros le traen. Un imperativo de buen trato es no molestarlos diciéndoles las verdades que los ofendan, por ejemplo: no decirles de su mal aliento o mirarlos feo por su tos persistente (Cfr. T. 589) ${ }^{11}$. A esta modalidad de comportamiento Hume añade un aspecto lúdico basado, además de la conversación, en el juego; esta instancia de la vida con los otros remite, también, a la idea de un 'progreso' en los sentimientos morales.

Sin embargo, a pesar de lo atractiva, la propuesta es porosa y se vacía por los huecos que deja el determinismo de los hábitos, las costumbres $\mathrm{y}$, muy especialmente, por la ruta ya trazada por las pasiones naturales, los hábitos personales y las costumbres culturales. La acción como volición de un agente fracasa ineludiblemente por sustrato de materia sentimental: en atención a (A1) un individuo egoísta no puede proponerse auto-transformarse en lo que no es, por muy noble y agradable que sea su objetivo.

\section{Fingir lo que no somos}

Una salida al determinismo es necesaria en cuanto corresponde a una de las cualidades más importantes que el ser humano presume, la autodetermación. Poseer buenas maneras y cooperar con las expectativas que otros tienen de él -tal como lo plantea Lewis- le permiten sentir las ventajas de reducir las pasiones egoístas, de conversar con los otros, de intercambiar sentimientos y, por esa vía, acordar comportamientos normativos posibles para el beneficio común ${ }^{12}$. Tal perspectiva manifiesta un adelanto en lo que

11 Esta actitud está implicando la aceptación de 'mentir por amor al prójimo', dejando de lado el proyecto de la autenticidad de la honestidad.

12 Besides, that we ourselves often change our situation in this particular, we every day meet with persons, who are in a different situation from ourselves, and who cou'd never converse with us on any reasonable terms, were we to remain constantly in that situation and point of view, which is peculiar to us. The intercourse of sentiments, therefore, in society and conversation, makes us form some general inalterable standard, by which we may approve or disapprove of characters and manners. And tho' the heart does not always take part with those general notions, or regulate its love and hatred by them, yet are they sufficient for discourse, and serve all our purposes in company, in the pulpit, on the theatre, and in the schools (T. 603). 
respecta a una moralidad basada en alguna modalidad de convenciones y/o en lo que el antiguo Derecho Romano llamaba ‘estipulaciones' surgidas por la conversación. El autor trató de resolver la limitación determinista por dos caminos, ambos propios de la facultad de la imaginación: por un lado, la concepción de la mente como un teatro; y por otro, la vía mágica (esotérica) por la cual el individuo se auto-percibe como alguien que puede, mediante un ritual mágico, realizar cambios categóricos en su ser. Ambos estarían en el campo de la autosugestión. Veamos.

\section{La mente como una puesta en escena}

La ficción juega un papel muy importante en la construcción de la moralidad. Para decirlo de alguna manera, la mente no percibe las cosas tal cual porque el mundo es variable o caótico. Para poder referirse a las cosas, la mente inventa la identidad y esta hay que configurarla como una puesta en escena. La única alusión que hace Hume a este respecto es importante pues refiere la mente como un teatro y la identidad de los objetos concebida solo por la imaginación del sujeto:

The mind is a kind of theatre, where several perceptions successively make their appearance; pass, re-pass, glide away, and mingle in an infinite variety of postures and situations. There is properly no simplicity in it at one time, nor identity in different; whatever natural propension we may have to imagine that simplicity and identity. The comparison of the theatre must not mislead us. They are the successive perceptions only, that constitute the mind; nor have we the most distant notion of the place, where these scenes are represented, or of the materials, of which it is compos'd (T. 253).

En lo que se refiere a la vida moral, uno de los aspectos más importantes de la filosofía de Hume es que la moralidad es un hecho mental e inexistente en el mundo externo ( $T$. 416-17), es un acto mágico ${ }^{13}$. Se trata de un hecho interno que implica una relación con un objeto ( $T .464-465,468-469)$ y que se realiza dentro de ese escenario teatral que termina siendo la mente. En el teatro, los personajes hacen creer al púbico que sienten las emociones de otros, representando situaciones que viven otros. Como los individuos no pueden ser agentes morales reales en la medida en que su ser en el mundo no coincide con sus proyecciones morales, el camino que les queda es representarse a sí mismos que son buenos, fingen ante la sociedad que poseen esos sentimientos;

13. La imaginación es una 'especie de facultad mágica del alma' y por tanto 'inexplicable para el entendimiento humano' (T.24). Ángela Calvo refiere, de manera correcta, a este ejercicio mental de creación del objeto de la siguiente manera:

[H] ume la describe (la imaginación) como una 'cualidad mágica de la mente' que vincula todas las comarcas de la geografía mental al conectar lo discreto de las percepciones en cadenas inerciales interminables, dando lugar a la experiencia con los objetos, así como a la interacción con los otros personajes del teatro del mundo... (Calvo 1998). 
el individuo se imagina ser de tal manera pero lo más importante, el otro también es imaginado así.

Sin embargo, lo que dice o hace una persona aun en el teatro de la mente no la transforma en eso. Al abordar el tránsito de la promesa al acto prometido, Hume recuerda al lector que una fórmula verbal no cambia las cosas, se mantiene, pues, fiel a lo planteado en el argumento (A1), en este caso al sujeto que las enuncia:

The difficulties, that occur to us, in supposing a moral obligation to attend promises, we either surmount or elude. For instance; the expression of a resolution is not commonly suppos'd to be obligatory; and we cannot readily conceive how the making use of a certain form of words shou'd be able to cause any material difference. Here, therefore, we feign a new act of the mind, which we call the willing an obligation; and on this we suppose the morality to depend. But we have prov'd already, that there is no such act of the mind, and consequently that promises impose no natural obligation (T. 523).

El mundo puede moverse como la puesta en escena de la sociedad justa y crear condición para que surja el gusto por la vida moral. El asunto es que vivir una 'perfomance teatral' es insuficiente en cuestiones morales. Debe haber algo que indique que la palabra no sea una mera emisión de sonidos vacíos (flatus vocis) y adquiera la dimensión de contenido proposicional y se relacione con una intención a la posibilidad de realización. La segunda salida no se relaciona con argumento alguno del empirismo, es mística y esotérica. Las acciones morales han de ser auténticas, deben salir del corazón del agente (T. 468-9). Esto corresponde al siguiente y último punto.

\section{La transmutación de la materia}

Este aspecto, el esotérico, muestra la dificultad que tuvo Hume para tratar de mostrar con coherencia que es posible la creación de los sentimientos morales basado en la idea de un determinismo de las pasiones. Como ya lo he afirmado, no es posible un cambio real en la conducta surgido del mero deseo del agente, pues esto es concluyentemente imposible ya que el cambio implica la adquisición por deseo del propio agente de 'cualidades diferentes' a las que naturalmente tiene. En relación con (A1) sería contradictorio:

A change of the obligation supposes a change of the sentiment; and a creation of a new obligation supposes some new sentiment to arise. But 'tis certain we can naturally no more change our own sentiments, than the motions of the heavens; nor by a single act of our will, that is, by a promise, render any action agreeable or disagreeable, moral or immoral; which, without that act, wou'd have produc'd contrary impressions, or have been endow'd with different qualities (T. 517).

La opción puede ser correcta si su mecanicismo o su fisicalismo no fuesen tan pronunciados y el objeto pudiera auto-transformarse. La salida, tal como Hume la presenta, es que entre la promesa (flatus vocis) como intención (intentio) y su realización de manera 
autónoma (actio libera), ha de suceder algo que no es natural sino milagroso ${ }^{14}$. Hume recurre a la analogía de la transustanciación propia de los ritos cristianos. La consagración y la elevación legitiman que un cristiano crea que el pan y el vino que va a consumir devocionalmente se hayan convertido en cuerpo y sangre de Cristo; de la misma manera, una promesa emitida públicamente por un individuo civilizado tiene que convertirse en el hecho prometido:

I shall farther observe, that since every new promise imposes a new obligation of morality on the person who promises, and since this new obligation arises from his will; 'tis one of the most mysterious and incomprehensible operations that can possibly be imagin'd, and may even be compar'd to transubstantiation, or holy orders, where a certain form of words, along with a certain intention, changes entirely the nature of an external object, and even of a human nature (T.524).

Esta salida podría ser válida en cuanto que el sentimiento moral no es un asunto de hecho. Ambos casos, la vivencia de roles a la manera del artista en escena y la modificación de la creencia por bases esotéricas corresponden a formas de fingimiento producto de la imaginación. El hecho podría traer buenos resultados en lo que concierne a una vida en unión con otros, pues garantizaría la reducción de las asperezas en las relaciones propias de individuos posesivos. Sin embargo, el asunto no es sencillo; un primer aspecto del problema que resulta es que, si bien el fingimiento puede ser un facilitador de relaciones sociales, no proporcionaría ningún cambio sustancial en los individuos de tal manera que garantice el respeto entre ellos; fingir (to feign) sentimientos no trae cambios reales en la conducta humana y con ello la condición pícara de los seres humanos no puede reducirse y, menos aún, desaparecer: es un comportamiento falso ${ }^{15}$. Esto constituye un problema moral, porque con esa actitud el individuo renuncia a su objetivo: tener sentimientos morales, a ser auténtico en su sentir respecto de las virtudes que lo definen como un ser moral.

Un segundo aspecto es que siendo coherentes con la tendencia que tienen los seres humanos a llevar un comportamiento autointeresado en concordancia con su sagacidad y su capacidad de fingir, una conducta fingida encajaría perfectamente con un buen trato estratégico hacia los otros ( $T 577,618$ y 218$)$ en la vida diaria y en los negocios. Los esfuerzos que una persona hace para sostener un ambiente de convivencia tienen carácter de virtud, porque cada cual debe limitar sus manifestaciones egoístas hacia los otros y conceder cosas que no acepta sinceramente. El argumento final de Hume

14 Años más tarde, en la primera investigación, Hume refiere a los milagros como una violación de las leyes de la naturaleza y por tanto no son susceptibles de veracidad: “"That no testimony is sufficient to establish a miracle, unless the testimony be of such a kind, that its falsehood would be more miraculous, than the fact, which it endeavours to establish..." (Hume 1999/1777, pp. 114-16) ¿A qué se debió este cambio en la teoría?

15 En A Dictionary of the English Language (1735). Su coetáneo Samuel Johnson definió el verbo fingir de la siguiente manera: "To FEIGN, -v. n. To relate falsely; to image from the invention; to tell fabulously" (p. 722). 
es que si el individuo acoge con gusto las ventajas que trae esforzarse por cumplir las promesas, tratar bien a los otros, respetar sus propiedades podría ser el inicio de algún cambio posible: "If we thought, that promises had no moral obligation, we never shou'd feel any inclination to observe them" $(T 518)^{16}$. La educación y el artificio de los políticos tienen esa función social: avivar las virtudes civilizadoras.

\section{La frustración}

A mi parecer, la teoría del fingimiento fracasa y puede valorarse como una forma de admitir la dificultad natural de lograr verdaderos sentimientos morales. Lo que espera Hume es que de tal proceso de autosugestión pudiera engendrar un nuevo acto de la mente que, a su vez, podría tener la fuerza obligatoria de la auto-convicción o sea alcanzar la importancia $\mathrm{D}^{2}$, ahí estaría su importancia ${ }^{17}$. Sin embargo, independientemente de la capacidad coactiva de la educación, la inclusión del fingimiento solo confirma una de sus posiciones más radicales planteadas en (A1): no se puede derivar un debe de un es.

Por un lado, son los otros los que finalmente determinan si el individuo en cuestión es merecedor de alabanza o repudio. Independientemente de lo que internamente él sienta, los efectos de sus esfuerzos son sociales, una buena 'apariencia' del individuo interesado contribuiría a aplacar los ánimos de los otros y a mostrar un camino agradable en las relaciones con el otro. La exigencia de respeto al otro también se encuentra reducida por prácticas de vinculación simpatética que proporcionan el hecho de compartir la mesa, el juego y conversar con él. Quizás la propuesta sea ingenua, porque no considera que haya algunos aspectos de difícil discusión, pero a mi leal saber y entender nadie se sentaría a negociar con alguien que solo realiza desplantes.

En consecuencia, la posibilidad de proyectar nuestras acciones a partir de la ejecución de virtudes morales, siguiendo su estructura humeana de la acción, sobrepasa las cualidades impuestas por los surcos que determinan lo que puede sentir la mente humana y por ende las actitudes del agente.

16 Esto es claramente coherente con su 'máxima metafísica' que afirma que lo que podemos imaginar claramente tiene una posibilidad de existencia: "[T] hat whatever the mind clearly conceives, includes the idea of possible existence, or in other words, that nothing we imagine is absolutely impossible" ( $T$ 32).

17 Resulta importante en este aspecto el trabajo de Willard Clark Gore, The Imagination in Spinoza and Hume. Gore resalta el hecho de que, según nuestro autor, la imaginación puede excitar las pasiones (Gore 1902). 
Referencias bibliográficas

Axelrod, R. (1984), The Evolution of Cooperation. New York: Basic Books.

Bentham, J. (2008), Los principios de la moral y la legislación. (M. Costa, Trans.) Buenos Aires, Argentina: Claridad.

Calvo, Á. (1998), Conversaciones y reciprocidad, El otro imaginado. Universitas Philosophiae 31: 11-29.

Ekman, P. (1996), Why Dont We Catch Liars? (N. I. Helth, Ed.), Social Research 63: 801-817.

Frankfurt, H. G. (1988), The Importance of What we Care About. New York: Cambridge University Press.

Gauthier, D. (1990), Moral Dealing. New York: Cornell University Press.

Glossop, R. J. (1976), Is Hume a 'Classical Utilitarian'? (H. Society, Ed.) Hume's Studies 2 (1): 1-16.

Gore, W. C. (1902), The Imagination in Spinoza and Hume. Chicago, Illinois USA.: Universidad de Chicago.

Hobbes, T. (1996/1651), Leviathan. (E. w. Gaskin, Ed.) New York: Oxford University Press.

Huff, T. (1972), Self-Interest and benevolence in Hume's Account of Moral Obligation. Ethics 1 (83): 58-70.

Hume, D. (1978), A Treatise of Human Nature. (L. A.-B. Edition, Ed.) England: Oxford, Clarendon Press.

(1999/1777), Enquiries Concerning the Human Understanding and Concerning the Principles of Morals. New York: Cambridge University Press.

Lewis, D. (1996). Convention: a philosophical study. Cambridge: Harvard University Press.

MacPherson, C. B. (1962), The Political Theory of Possessive Individualism. New York: Oxford University Press. 